\title{
ChemComm
}

\section{Potassium formate as a small molecule switch: controlling oxidation-reduction behaviour in a two-step sequence $\dagger$}

Cite this: Chem. Commun., 2013
49, 3143
Received 6th January 2013,
Accepted 21st February 2013

DOI: $10.1039 /$ c3cc00126a

www.rsc.org/chemcomm

A one-pot reaction sequence is described in which the addition of one compound allowed switching between simultaneously occurring oxidation and reduction reactions.

In the past decade, multi-step one-pot procedures have attracted increasing interest of the chemical community. ${ }^{1}$ Combining multiple transformations in one reaction vessel renders sequences more costeffective as fewer work-up and purification steps are required. Multistep one-pot procedures are therefore considered to contribute to a more sustainable way of producing chemicals. A major challenge in the design of multi-step sequences is the compatibility of the involved substrates, intermediates and reagents or catalysts.

A particular challenge is seen in reactions in which opposing or counteracting reagents or catalysts (acid-base, oxidator-reductor) are used simultaneously. ${ }^{2}$ Incompatibilities between opposing catalysts have been resolved by immobilization on insoluble polymers $^{3}$ or encapsulation in sol-gels. ${ }^{4}$ Such behaviour is advantageously used in for example dynamic kinetic resolutions and related procedures $^{5}$ in which secondary alcohols are racemised via the corresponding ketones through a redox equilibrium induced by a single catalyst. A remarkable case has been described by Kroutil et al., in which racemisation and reduction were performed stepwise using a single alcohol dehydrogenase (ADH). Switching between non-selective oxidation and enantioselective reduction occurred by removal of the co-substrate acetone and addition of i-propanol. ${ }^{6}$

In a different strategy, enantioselectivity has been achieved via chemical oxidation by a metal-catalyst, and simultaneous reduction by $\mathrm{ADH}$ in the same reaction vessel. ${ }^{7}$ As shown in this approach, performing multistep reactions in a consecutive manner does not per se require the adaptation of the catalysts or reactants used.

In this communication, we describe a model one-pot reaction system with counteracting catalysts, in which the oxidant acts on the starting compound and the final product, while the reductant

Institute for Molecules and Materials, Radboud University Nijmegen, Heyendaalseweg 135, 6525 AJ NIjmegen, The Netherlands.

E-mail: F.Rutjes@science.ru.nl; Fax: +31 243653393; Tel: +31 243653202

$\dagger$ Electronic supplementary information (ESI) available. See DOI: 10.1039/ c3cc00126a

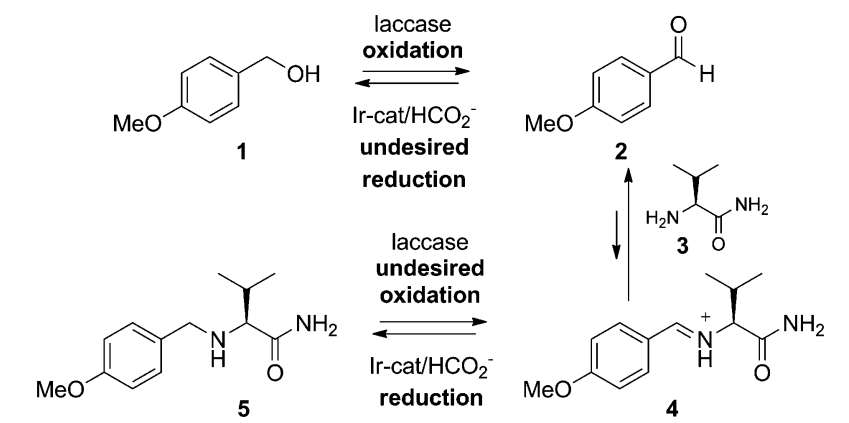

Scheme 1 One-pot oxidation-reduction sequence.

acts on the intermediate product leading to both the starting material and the final product. We have shown that by introducing a specific reagent, the reaction can be efficiently steered into one direction by 'switching-off' the oxidation and simultaneously 'switching on' the reduction, with all catalysts present from the start of the reaction. This is the first example of a reagent-mediated switch between two counteracting catalytic systems, which can now be efficiently performed in one pot in the desired sense.

The two-step sequence involves the aqueous enzymatic oxidation of $p$-methoxybenzyl alcohol (1) to anisaldehyde (2), which in the presence of valinamide (3) is in equilibrium with iminium intermediate 4 (Scheme 1). For the enzymatic oxidation laccase was selected because of its outstanding oxidative performance on benzylic systems and its broad substrate selectivity. ${ }^{8}$ The iminium ion 4 reacts further in an iridium-catalyzed reductive amination with formate as the hydride source to yield amine $\mathbf{5}$. However, since the latter product is readily oxidized by laccase, and benzaldehyde is also prone to reduction by the iridium catalyst, this process does not lead to any product formation when carried out in a single vessel. The major challenge therefore was to limit the undesired reverse reactions.

In order to tackle the problem, we initially analysed the partial reactions involved in more detail. Laccase belongs to the class of oxidoreductases and is capable of performing oxidations at the expense of $\mathrm{O}_{2}$ with water as the only byproduct. Because of its stability and non-toxic waste, laccase is also widely applied in industry. ${ }^{9}$ Laccase readily oxidised anisyl alcohol (1) to aldehyde 2 


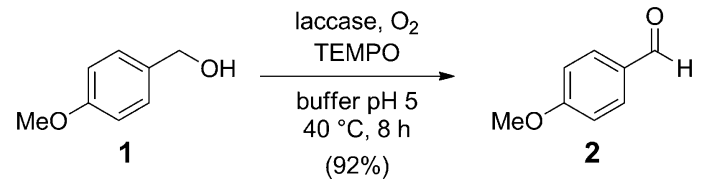

Scheme 2 Laccase-mediated oxidation of anisyl alcohol (1).

in oxygenated citric acid buffer at pH 5 in 92\% yield (Scheme 2). We selected an activated alcohol $^{10}$ to keep the reactions short. In addition, 0.3 equivalents of TEMPO were used as an electron carrier, which appeared crucial for the oxidation to proceed. ${ }^{10,11}$

The reducing agent in our sequence was iridium complex 6 (Scheme 3), which has been successfully applied in aqueous reductive amination reactions. ${ }^{12}$ The catalyst is based on related iridium complexes which catalyse transfer hydrogenation reactions as has been documented by Fukuzumi et al. ${ }^{13}$ The reductive amination of 2 with (S)-valinamide (3) to give the stable product 5 proceeded in $92 \%$ yield under similar conditions in buffer at pH 5 at $40{ }^{\circ} \mathrm{C}$ (Scheme 3). Thus, iridium complex 6 was capable of reducing the intermediate iminium ion 4 in the presence of $\mathrm{HCO}_{2} \mathrm{~K}$ as the hydride source. An excess of anisaldehyde (2) was used, because it was partially reduced to anisyl alcohol (1) in a competing reaction.

Next, the compatibility of the oxidation conditions with each of the reactants of the reductive amination was examined (Table 1). The presence of either valinamide (3, entry 2 ) or iridium complex 6 (entry 3) was tolerated. Contrarily, a $64 \mathrm{mM}$ concentration of $\mathrm{HCO}_{2} \mathrm{~K}$ (1 equiv.) almost completely inhibited the enzymatic activity, while $32 \mathrm{mM} \mathrm{HCO}_{2} \mathrm{~K}$ ( 0.5 equiv.) resulted in only a small decrease in yield. To investigate whether this inhibition is reversible, we removed $\mathrm{HCO}_{2} \mathrm{~K}$ by filtration over a spin-filter, after which the recovered laccase was tested in the oxidation of alcohol 1. Formation of aldehyde $\mathbf{2}$ was indeed observed, showing that laccase is only reversibly inactivated by $\mathrm{HCO}_{2} \mathrm{~K}$.

The same approach was used for the reductive amination (Table 2), which revealed that the reaction was not hampered by TEMPO (entry 2). The presence of laccase (entry 3) did have a

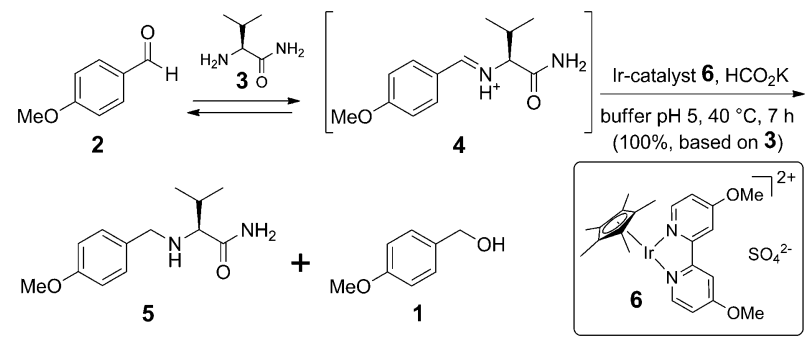

Scheme 3 Aqueous reductive amination of anisaldehyde (2).

Table 1 Compatibility of the enzymatic oxidation with the reactants of the reductive amination ${ }^{a}$

\begin{tabular}{lll}
\hline Entry & Additive & Conversion after $8 \mathrm{~h}^{b}(\%)$ \\
\hline 1 & - & 92 \\
2 & Valinamide (3) (0.66 equiv.) & 91 \\
3 & Iridium complex 6 (5 mol\%) & 96 \\
4 & $\mathrm{HCO}_{2} \mathrm{~K}(64 \mathrm{mM})$ & 2 \\
5 & $\mathrm{HCO}_{2} \mathrm{~K}(32 \mathrm{mM})$ & 84
\end{tabular}

${ }^{a}$ Conditions: 25 U laccase, 0.3 equiv. TEMPO. ${ }^{b}$ Conversions determined by RP-HPLC.
Table 2 Compatibility of the reductive amination with the reactants of the oxidation ${ }^{a}$

\begin{tabular}{lll}
\hline Entry & Additive & Conversion after $8 \mathrm{~h}^{b}(\%)$ \\
\hline 1 & - & 100 \\
2 & TEMPO (0.3 equiv.) & 100 \\
3 & Laccase (25.0 U) & 64 \\
4 & Laccase (25 U) + TEMPO (0.3 equiv.) & 3
\end{tabular}

${ }^{a}$ Conditions: 5 equiv. 2 , 10 equiv. $\mathrm{HCO}_{2} \mathrm{~K}, 0.05$ equiv. 6. ${ }^{b}$ Conversions determined by RP-HPLC.

tolerable negative influence on the yield. The work of Arends et al. revealed that the rhodium analogue of complex 6 shows a significant decrease in activity in the presence of various enzymes due to complexation of the metal center. ${ }^{14}$ In our hands the activity loss was limited, because a higher complex/enzyme ratio was used. In the presence of both laccase and TEMPO (entry 4), an initial yield of $8 \%$ was observed after $4 \mathrm{~h}$, which gradually decreased to provide only traces of product 5 after $7 \mathrm{~h}$, indicating that the product had reacted with another compound.

Fabbrini and coworkers showed that benzylic amines can be oxidized to iminium ions by the oxidized TEMPO species, resulting in the corresponding aldehydes in aqueous media. ${ }^{11 b}$ Likewise, the alkylated valinamide $\mathbf{5}$ may be rapidly oxidized via the iminium intermediate to aldehyde $\mathbf{2}$ and valinamide $\mathbf{3}$ in the presence of laccase and TEMPO. A competition experiment with alcohol $\mathbf{1}$ and amine $\mathbf{5}$ confirmed that the amine is more easily oxidised at $\mathrm{pH} 5.0$ to aldehyde 2 than anisyl alcohol (1) (Scheme 4). At the time amine 5 was fully oxidised to aldehyde 2, 63\% of anisyl alcohol (1) was still left. Competitive experiments at lower $\mathrm{pH}$ also gave a higher rate for the amine oxidation $(\mathrm{pH} 4.5-3.5)$ or resulted in laccase inactivation (pH 3.0-2.5). †

The latter results underline that it is impossible to conduct both transformations simultaneously, and that in a one-pot system the products revert to the starting materials. We then chose to adjust the procedure, prompted by the observed inhibition of laccase by $\mathrm{HCO}_{2} \mathrm{~K}$ (Table 1 , entry 4 ). To test whether laccase could be inactivated and oxidation of amine $\mathbf{5}$ would be suppressed, the reductive amination was performed in the presence of laccase, TEMPO and 25 equivalents of $\mathrm{HCO}_{2} \mathrm{~K}$. Indeed, $88 \%$ of the desired product was formed, showing that $\mathrm{HCO}_{2} \mathrm{~K}$ could be used as a switch between the oxidation and the reduction.

After establishing the possibility to switch between the two different reactions, we set out to optimize the one-pot oxidationreduction process (Scheme 5). Reaction in the absence of laccase (Table 3, entry 1) did not lead to any product formation. Without

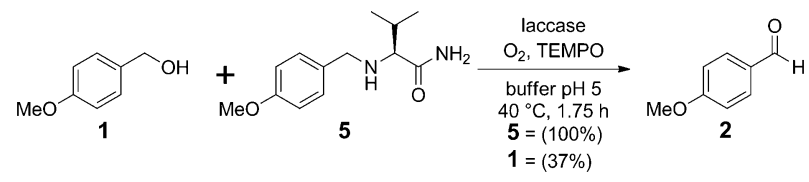

Scheme 4 Competitive oxidation of amine 5 and alcohol 1

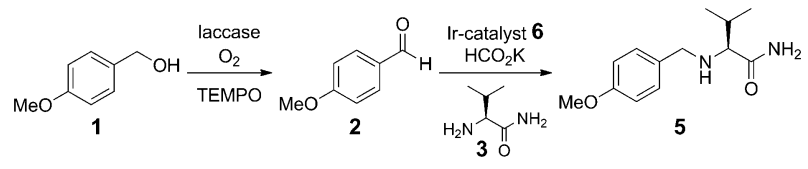

Scheme 5 One-pot two-step sequence with opposing catalysts. 
Table 3 One-pot two-step sequence optimization

\begin{tabular}{|c|c|c|c|c|c|c|c|}
\hline Entry & Laccase (U) & Ir-cat (mol\%) & $\mathrm{HCO}_{2} \mathrm{~K}$ (equiv.) & Time for oxidation $(\mathrm{h})$ & Alcohol conversion $^{a, b}$ (equiv.) & Total reaction time $(\mathrm{h})$ & Conversion $^{a}(\%)$ \\
\hline 1 & 0.0 & 5 & 10 & 10 & 0.00 & 10 & 0 \\
\hline 2 & 25.0 & 5 & 0.5 & 24 & 5.00 & 24 & 0 \\
\hline 3 & 25.0 & 5 & 100 & 12.5 & 4.46 & 20 & 18 \\
\hline 4 & 25.0 & 5 & 25 & 12.5 & 4.61 & 20 & 29 \\
\hline 5 & 2.50 & 5 & 10 & 13 & 4.44 & 20 & 32 \\
\hline 6 & 2.50 & 5 & $2 \times 2.5$ & 16.5 & 4.32 & 65 & 64 \\
\hline 7 & 2.50 & 1 & 10 & 22 & 3.67 & 50 & $80(40)^{c}$ \\
\hline 8 & 2.50 & 0.5 & 10 & 23 & 3.78 & 71 & 70 \\
\hline 9 & 2.50 & 0.2 & 10 & 17 & 4.50 & 136 & 61 \\
\hline 10 & 2.50 & 1 & $3 \times 2.5$ & 23 & 3.78 & 88 & 81 \\
\hline $11^{d}$ & 2.50 & 1 & 10 & 41 & 3.51 & 65 & $91(50)^{c}$ \\
\hline $12^{e}$ & 2.50 & 1 & 10 & 41 & 2.36 & 41 & $78(62)^{c}$ \\
\hline
\end{tabular}

${ }^{a}$ Conversions determined by RP-HPLC. ${ }^{b}$ With respect to valinamide $3 .{ }^{c}$ Isolated yields are given in brackets. Isolated yields were lower due to losses during the extraction procedure. ${ }^{d}$ Two times more concentrated. ${ }^{e}$ Four times more concentrated.

inhibition of laccase - in the presence of small amounts of $\mathrm{HCO}_{2} \mathrm{~K}-$ no desired product was formed either (entry 2). If 100 equivalents of $\mathrm{HCO}_{2} \mathrm{~K}$ were added, laccase was successfully inhibited (entry 3) albeit that only $18 \%$ of the desired compound was obtained. The low yield was the result of a second, fast competing reduction, the aldehyde being reduced to alcohol 2 . The iminium ion is formed in an unfavourable equilibrium reaction in water, and a fast reduction mainly results in aldehyde reduction. Decreasing the reduction rate should afford more product 5 because more time is available to set the equilibrium. Indeed, when 25 equivalents of $\mathrm{HCO}_{2} \mathrm{~K}$ were used (entry 4), the yields significantly increased. A further enhancement was achieved by using 10 equivalents of $\mathrm{HCO}_{2} \mathrm{~K}$ (entry 5 ). In this case, a ten-fold lower amount of laccase was used as otherwise the enzyme would not be inhibited efficiently and oxidation of compound $\mathbf{5}$ would occur. Remarkably, the oxidation of the alcohol was only slightly slower in entry 5 compared to that in entries $2-4$. When $\mathrm{HCO}_{2} \mathrm{~K}$ was added in two portions of 2.5 equivalents a yield of $64 \%$ was observed. To summarize, by using the appropriate amount of the 'switching' compound, the yield was increased from traces to $64 \%$.

We continued the optimisation by varying the amount of catalyst 6 (entries 7-9). The presence of 0.5 or $1 \mathrm{~mol} \%$ catalyst gave high yields of 70 and $80 \%$, respectively, while $0.2 \mathrm{~mol} \%$ catalyst led to a disagreeable reaction time of $136 \mathrm{~h}$ and a lower yield. In entry 10, optimised amounts of both $\mathrm{HCO}_{2} \mathrm{~K}$ (2.5 equiv.) and iridium complex 6 (1 mol\%) were used. However, a comparable yield was obtained in the example in which $\mathrm{HCO}_{2} \mathrm{~K}$ was added in one batch (entry 7).

A last strategy involved using more concentrated reactions, thereby increasing the concentration of iminium ion 4 . Indeed, a high yield of $91 \%$ (entry 11) was obtained if the reaction was performed twice as concentrated. Contrarily, 78\% (entry 12) was found with a four times more concentrated reaction mixture. In the latter case, the enzymatic oxidation appeared to be slow and far from completion, explaining the lower yield. Probably the high concentration of reagents hampered the laccase activity.

Preliminary experiments to apply this switch concept to substituted benzylic alcohols indicated that in each of these cases extensive experimentation was required, which is considered to be beyond the scope of this communication.

A successful two-step reaction sequence with counteracting catalysts in water was performed. An enzymatic oxidation was combined with a reducing metal catalyst in one-pot. First, anisyl alcohol (2) was oxidised to anisaldehyde (3), which reacted in a reductive amination with valinamide (4). The reaction sequence was performed subsequently in which it was crucial to inhibit the laccase with an excess of $\mathrm{HCO}_{2} \mathrm{~K}$. At the same time, the reductive amination started. The reaction conditions were fine-tuned and led to a maximum conversion of $91 \%$ and a maximum isolated yield of $62 \%$. This is the first example in which a compound acts as a switch between two opposing reactions in a one-pot procedure.

\section{Notes and references}

‡ Details of the competitive oxidation under various $\mathrm{pH}$ values can be found in the ESI. $†$

1 (a) A. Bruggink, R. Schoevaart and T. Kieboom, Org. Process Res. Dev., 2003, 7, 622-640; (b) J. Zhou, Chem.-Asian J., 2010, 5, 422-434.

2 J. H. Schrittwieser, J. Sattler, V. Resch, F. G. Mutti and W. Kroutil, Curr. Opin. Chem. Biol., 2011, 15, 249-256.

3 B. J. Cohen, M. A. Kraus and A. Patchornik, J. Am. Chem. Soc., 1981, 103, 7620-7629.

4 (a) F. Gelman, J. Blum and D. Avnir, Angew. Chem., Int. Ed., 2001, 40, 3647-3649; (b) F. Gelman, J. Blum and D. Avnir, New J. Chem., 2003, 27, 205-207; (c) S. L. Poe, M. Kobašlija and D. T. McQuade, J. Am. Chem. Soc., 2006, 128, 15586-15587.

5 (a) O. Pàmies and J. Bäckvall, Chem. Rev., 2003, 103, 3247-3261. For some examples, see $e . g$; (b) A. L. E. Larsson, B. A. Persson and J. Bäckvall, Angew. Chem., Int. Ed., 1997, 36, 1211-1212; (c) D. Cartigny, K. Puntener, T. Ayad, M. Scalone and V. Ratovelomanana-Vidal, Org. Lett., 2010, 12, 3788; (d) J. Limanto, S. W. Krska, B. T. Dorner, E. Vazquez, N. Yoshikawa and L. Tan, Org. Lett., 2010, 12, 512.

6 C. V. Voss, C. C. Gruber and W. Kroutil, Tetrahedron: Asymmetry, 2007, 18, 276-281.

7 F. G. Mutti, A. Orthaber, J. H. Schrittwieser, J. G. de Vries, R. Pietschnig and W. Kroutil, Chem. Commun., 2010, 46, 8046-8048.

8 (a) S. Riva, Trends Biotechnol., 2006, 24, 219-226; (b) S. Witayakran and A. J. Ragauskas, Adv. Synth. Catal., 2009, 351, 1187-1209; (c) J. M. M. Verkade, L. J. C. van Hemert, P. J. L. M. Quaedflieg, H. E. Schoemaker, M. Schürmann, F. L. van Delft and F. P. J. T. Rutjes, Adv. Synth. Catal., 2007, 349, 1332-1336.

9 (a) S. Rodríguez. Couto and J. L. T. Herrera, Biotechnol. Adv., 2006, 24, 500-513; (b) A. P. Virk, P. Sharma and N. Capalash, Biotechnol. Processes, 2012, 28, 21-32.

10 M. Fabbrini, C. Galli and P. Gentili, J. Mol. Catal. B: Enzym., 2002, 16, 231-240. 11 (a) A. Potthast, T. Rosenau, C. L. Chen and J. S. Gratzl, J. Mol. Catal. A: Chem., 1996, 108, 5-9; (b) M. Fabbrini, C. Galli, P. Gentili and D. Macchitella, Tetrahedron Lett., 2001, 42, 7551-7553.

12 J. M. McFarland and M. B. Francis, J. Am. Chem. Soc., 2005, 127, 13490-13491.

13 (a) T. Abura, S. Ogo, Y. Watanabe and S. Fukuzumi, J. Am. Chem. Soc., 2003, 125, 4149-4154; (b) S. Ogo, K. Uehara, T. Abura and S. Fukuzumi, J. Am. Chem. Soc., 2004, 126, 3020-3021; (c) S. Ogo, K. Uehara, T. Abura, Y. Watanabe and S. Fukuzumi, J. Am. Chem. Soc., 2004, 126, 16520-16527. 14 M. Poizat, I. W. C. E. Arends and F. Hollmann, J. Mol. Catal. B: Enzym., 2010, 63, 149-156. 